\title{
THE OPERATIVE TREATMENT OF ENLARGED PROSTATE.
}

\author{
By ARTHUR CHANCE, F.R.C.S.; \\ Surgeon to the Mater Misericordiæ Hospital, Dublin ; \\ Surgeon to the Dublin Orthopædic Hospital. \\ [Read in the Section of Surgery, December 8, 1893.]
}

THE frequency of obstructive prostatic enlargement, the harassing character of the disease, and the inadequate nature of its palliative treatment will, I hope, justify me in bringing the subject before you to-night. And that I may have a text to act as a base on which to build my remarks, I will read to you brief notes of a case in which I performed prostatectomy with a fair measure of success.

CASE.-My patient was a worn-looking farm labourer, aged fifty-six. Save for an occasional cold, the result of exposure, he had had no serious illness, and prior to September, 1891, he had never suffered from any form of genito-urinary disorder. At that time he noticed a gradually increasing difficulty in making water. Micturition became more frequent, the stream weaker, and pain was felt over the pubes. Defæcation was accomplished with much straining, and the motions were flattened.

In February, 1892, these symptoms culminated in an attack of complete retention, and for this condition he was first admitted to the "Mater."

On examining through the rectum the prostate was found to be very large, so much so that it was difficult to pass the finger into the bowel. The growth was of elastic hardness, resembling to the touch solid India-rubber.

After catheterism and other appropriate treatment had been adopted for some days the power of voluntary micturition returned, but the act was still difficult, painful, and very frequent.

With the idea of reducing the hypertrophy I determined to try the effect of electrolysis. I, therefore, had a curved trocar made, 
insulated, save for about half an inch at its point. After emptying the bowels, washing out the rectum with an antiseptic solution, and dilating the sphincter, $I$ introduced the point of the trocar well into the prostatic mass. On account of the size and fixity of the growth this procedure was easy; but it was accompanied by free hæmorrhage. Connecting the trocar with the negative pole of a Leclanche battery I completed the current by applying to the inner side of the thigh a large charcoal electrode connected with the positive pole. The current was then permitted to flow for about fifteen minutes when it was for a short time reversed. This procedure was repeated six times at intervals of from seven to fourteen days.

At the conclusion of this treatment the patient could hold water for about an hour at a time. Pain and straining still continued.

I lost sight of my patient for some months, but on the 13th December, 1892, he again appeared in a condition much worse than before. He was worn and haggard looking. The straining had increased although the stream was weaker; the pain was worse, and the intervals between the acts of micturition were only of about ten minutes duration. To use his own graphic phrase" he hadn't time to warm the bed before he had to get out again," and his distress was so great that "he was praying for a man to come and shoot him." 'The residual urine was only about two ounces, although the patient thought his bladder nearly full. The water contained neither pus, albumen, nor sugar. Defæcation was still diflicult.

On the 24th January, 1893, I proceeded to perform supra-pubic prostatectomy. The bladder was repeatedly washed out with warm boracic solution and was then as far as possible emptied through a catheter. Subsequently a quantity of about fourteen ounces of boracic solution was introduced by means of a syphon from a vessel held about four feet from the operating table. The penis was then ligatured. No rectal bag was used. As I laid my hand on the relaxed parietes I was surprised to find how thoroughly the bladder was brought into contact with the abdominal wall. An incision of about two and a half inches was made and the bladder exposed. It was seized with a sharp hook, incised to the extent of about an inch and a half, and the edges of the incision were secured by ligatures passed through the bladder wall and held at their free ends by an assistant. The inner surface of the 
bladder seemed healthy. The prostate projected inwards as a large hard mass, not unlike the cervix uteri. The bladder was considerably depressed below this ingrowth. The opening of the urethra could not be detected until a sound had been introduced. It was then found at the apex of the cone-shaped projection. I found it difficult to use a scissors effectively for removing the growth. Instead I used a flushing scoop connected with an irrigator filled with sterilised hot water. This apparatus I found most satisfactory. Guided by the sound I scooped out a funnelshaped opening along the track of the urethra. The hæmorrhage was alarming, filling the bladder, and being washed out in large clots by the strong stream of water; but it was stopped rather quickly by flushing with hot water. The prostatic tissue was tough and difficult to excavate. It was washed away in small pieces. I think, all told, I removed about half an ounce of the growth. I then passed my finger well into the prostatic urethra and found no obstruction.

The upper portion of the parietal incision was closely sutured, and the bladder stitched to the abdominal wall. No drainage tube was used.

Briefly, the man had not a bad symptom. He commenced to pass water through the urethra on the twentieth day after operation, and the wound was healed on the forty-second. I showed him at the June meeting of the Academy. He could then hold water for seven hours.

In August he was engaged in building hayricks, and when thrusting up the hay from the ground he slightly strained the abdominal cicatrix. There was no hernia, merely a weak spot. I directed him to wear a suitable truss.

On December 2nd, that is more than ten months after the operation, I again examined him. His health was much improved and he had no pain or trouble of any kind. He could hold water easily for ten or eleven hours, and he assured me that for months he had not found it necessary to micturate during the night. After a long night he found it difficult but not impossible to empty his bladder completely at the first effort. It was easier to partially empty it first and a few minutes later to complete the act. He passes a fair sized stream of less than normal force without straining. Difficulty of defæcation still exists, but is somewhat diminished. 
Some points in this case merit comment. The large prostates which give iise to bladder trouble rarely project into the rectum. In this case, as I have mentioned, there was much straining at stool, and there was even some difficulty in introducing the finger. The large size of the prostate and the extent to which it was fixed in the pelvis forbade the use of a rectal bag during the operation; but, as I have said, a bag was not necessary.

The "uniform circular projection surrounding the internal orifice of the urethra," as M“Gill terms the condition found in my case, is not an unusual form of prostatic enlargement. Its frequency has been overlooked from the fact that when at a post-mortem examination on such a case the urinary organs are removed from the body, the prostate, losing the support of the surrounding tissues, does not project into the bladder as it did during life.

The influence of this form of enlargement in causing. partial retention of urine is ingeniously compared by $M$ 'Gill to the action of the mitral valve. In the heart when the ventricle contracts the valve is closed. When the bladder forcibly contracts the intravesical pressure squeezes the valve-like sides of the prostatic projection together so as to more or less completely close the urethra. When the violent effort to urinate ceases the greater part of the water gradually flows away.

It appears to me that the usual method of estimating: the amount of residual urine is open to fallacy. Does the passage of a catheter in a patient such as mine completely empty the bladder? The expulsive force of the bladder is usually impaired, but, apart from this, a considerable quantity of the urine, distant from the eye of the catheter, must collect round the projecting prostate. Indeed, the condition of the bladder resembles one of these "buttonhole" "non-spilling" ink bottles, from which the ink 
cannot be emptied completely, no matter in what position the bottle may be placed.

Possibly this may be the explanation of the small amount of residual urine drawn off from my patient. Except on some such supposition I find it difficult to explain that with only about two ounces of residual urine (as usually estimated) and with no well-marked inflammation, micturition could have been so persistently frequent.

I am inclined to attribute the good result following: electricity more to the free hæmorrhage than to any electrolitic action.

Three routes have been suggested for getting at the enlarged prostate-the supra-pubic, the urethral, and the perineal. That the supra-pubic is the most effective is too obvious a proposition to need defence. But without suture of the bladder, and this is impracticable in most cases, supra-pubic prostatectomy is open to two disadvantages-inefficient drainage and a yielding abdominal cicatrix. As regards the first, my opinion is, I know, at variance with that held by many surgeons. But it seems to me that as the patient lies on his back the bladder overfluws rather than drains through the supra-pubic wound. No doubt, the abdominal viscera compress the bladder so as to minimise its capacity, but a channel must exist between the ureters and the abdominal opening, and this channel running past the retro-pubic space is constantly full of urine. Besides the raw cavity of the prostate cannot but be unfavourably affected by the lodgment of irritating urine.

M'Gill held that supra-pubic drainage was efficient, but in the same paper in which he formulates this view, he mentions three cases of suppuration in the retro-pubic space following supra-pubic cystotomy. 
It may be urged that by using a tube the danger of extravasation is minimised, but, as Mr. Treves says, "there is no object in introducing a tube into the bladder, and the various syphon drainage tubes are delusions and snares. - . No drainage tube will prevent the occasional escape of urine into the connective tissues around the incision."

That the supra-pubic incision alone does not effectively drain the bladder was well demonstrated by a case I had some time since. My patient was a clergyman on whom I performed supra-pubic lithotomy removing four fairly large stones. It follows that the opening in the bladder was of considerable size. My patient had long been a diabetic, and the disease seemed re-kindled by the operation, as for some time afterwards he had intense thirst and passed large quantities of urine; yet, in spite of all this, for the first eight days every drop of urine came by the urethra.

Having regard to the possibility of a yielding cicatrix, I would deprecate the transverse division of any of the structures forming the abdominal wall. The upper end of the parietal wound should, of course, be carefully sutured. I cannot but think that the leaving open for drainage purposes of a part of the abdominal wound must occasionally result in insecure union.

In my case the exertion caused by lifting bundles of hay was the exciting cause of the yielding of the cicatrix; but the straining at stool was probably a contributing factor.

It seems to me that perineal drainage is the best way to avoid both the difficulties of which I have spoken It would give efficient drainage, and in this way would facilitate the early closure of the abdominal wound. The perineal opening would also permit of the free exploration 
of the prostatic urethra and the detection and removal of grow ths that might not be obvious to the finger introduced from within. In addition the effect of a large tube in moulding the new prostatic path might be of distinct value.

I would advocate, therefore, the introduction through a median perineal incision of a large flanged drainage tube such as is used in empyema, but with no side openings save near the inner end. The urine could be caught by a carbolised sponge as in perineal lithotomy. The use of a tube leading directly from the bladder to a vessel outside the bed is open to the objection that such a tube may be readily obstructed either by kinking or by pressure from without.

Of course a perineal section means some added risk, but I think the advantages to be gained outweigh this. The possible formation of a permanent fistula is another difficulty, but Wishard's operation might be relied on to close this.

Passing to a broader question, has not the time come to advocate the earlier performance of prostatectomy? The obstruction is due to an organised hypertrophy which no palliative treatment will remove. It increases with time and the symptoms it causes are distinctly progressive.

Every day the bladder degenerates and the prospect of success after a radical operation diminishes. Inflammatory changes may occur despite all precautions. Complete retention with subsequent atony may result from but a little indiscretion. And at best is not catheter life a euphemistic name for a peculiarly irksome condition of slavery.

Excluding those whose condition forbids all operative procedure, are we in the remaining cases to adopt palliative measures until they break down, or are we to adopt 
prostatectomy at the earliest favourable moment after obstructive prostatic enlargement is with certainty diagnosed? It is to solve this question that I ask your aid.

Mr. Kendal Franiss congratulated Mr. Chance on the success attending him in this case. He himself had seen this case in the Mater Hospital. He had the satisfaction of knowing from the patient himself that there was a great improvement in his condition. He thought that this operation was one of the many operations that marked the progress of surgery in the latter part of the nineteenth century. They had all met with a great many cases of prostate enlargement, and what a miserable class of cases they were! They knew that after catheter-life had been established the patient's life was frequently simply unendurable. They accepted with great pleasure the recommendation of Sir H. Thompson for relieving this miserable condition a few years ago. This method of draining the bladder through the perinæum he had tried in several cases, and he saw that it was certainly a most valuable procedure for giving physiological rest to the bladder, and with relief of all symptoms for a time. But in this operation they had a much more valuable method of dealing with it, because they not only gave physiological rest but they also removed the cause of the disease. He entirely agreed with $\mathrm{Mr}$. Chance as to the value of supra-pubic drainage, but he thought where they had a healthy perinæum they should deal with it there, because it was putting the drainage in the most dependent position. Otherwise, the maximum amount of drainage we get is the overflow, and we do not get the bladder completely emptied. He suggested that in these cases of prostatectomy the best thing to do was to make, in addition, a perinæal opening and put in a big tube. In the suprapubic operation he thought it a very desirable thing in most cases to obtain primary union of the bladder wound, because they would not then have a weak spot in the abdominal walls, and it was the point where the drainage-tube was put in which frequently yielded, and where hernia was most likely to occur. As to the time at which the operation should be done, he thought that in suitable cases the earlier it was done the better. He thought there would be a far larger percentage of successes if they only took the thing 
in time. He would be inclined to ssy that once the catheter required to be passed more frequently than every second or third hour, the limit would be reached at which the operation should be adopted.

Mr. MYLES thought that this admirable paper presented a subject of the greatest importance and the greatest difficulty. His own experience was that the diagnosis of these cases was not at all simple. He instanced a case sent from the country as one of prostatic enlargement. The patient had all the ordinary symptoms, as difficulty of micturition, and on examination per rectum the prostate was found enlarged. All the ordinary catheters got stuck at the bladder, but a short-beaked staff entered The patient subsequently died, and his bladder was found to be absolutely normal, so that he thought it was a very difficult matter to know what cases of prostatic enlargement were cases for operation. He said if they examined the specimens in the Museum of the College they would find an extraordinary diversity in the types of prostatic enlargement. He remembered $\mathrm{Mr}$. Tobin reading an excellent paper on this subject two years ago, and he brought down to the meeting several interesting specimens. Some of the specimens were those of a pediculated tumour growing from the posterior part of the prostate, and absolutely excluding the aperture of the urethra. Others presented ragged, irregular enlargements of such a type that operations on the middle lobe would do no good. They could only be simply scraped away or cauterised. Another point of great importance in these cases was the fact that the tenure of life in these patients was extremely limited. He instanced the patient already referred to, who, three hours after the instrument was passed, got a severe rigor, which was repeated, and he died at ten o'clock that night. On examination, all the organs were healthy save the kidneys, which were in a state of hydronephrosis. He said, therefore, that if a man with an enlarged prostate should die after examining the bladder, how much more likely was he to die if he submitted to operation. He next referred to the electrical treatment of $\mathrm{Mr}$. Chance, and said that he himself had used it on his private patients, much to his own and their detriment. He would like to know whether Mr. Chance used the galvanometer, and whether an increase in the force of the current produced a corresponding diminution in the size of the tumour. With regard to supra-pubic drainage, he submitted there were means by which the bladder could be kept quite empty, 
He explained how by means of an Indian-rubber apparatus a perfect syphon would be formed by which not a drop of urine would be allowed to collect in the bladder; and the kinks could be avoided also. He thought it a very difficult matter to tap the bladder through the perinæum if the prostate be enlarged. The distance between the perinæum and the floor of the bladder is fully three inches, and it was not every practitioner's fingers that could reacb it. He also wished to question the use of the term "residual urine." Were they to understand by that the urine that remains after the patient has exerted all his voluntary efforts, or after the surgeon has evacuated what he can by the catheter. He did not think that the mechanism of the catheter was properly understood, because it was immaterial whether the catheter was placed above the actual level of the fluid or below it, as the fluid would flow in the line of least resistance owing to the pressure inside the abdomen being always positive.

Mr. Chance, in reply to Mr. Franks, said with regard to the closing of the bladder, that the probability of failure would be very much dimmished if perinæal drainage were employed, because there would then be no strain on the bladder. With regard to the time of the operation, when there was obstructive prostatic enlargement, he did not see the use of wasting time but would deal with it at once. He admitted with Mr. Myles that the difficulty of diagnosis was great, but he always adopted the precaution of first reducing any inflammation by mild measures, and he thought that after that the age, symptoms, \&c., would lead them to a right conclusion. With regard to the different kinds of growth, he said his case was of a most difficult kind, because it was a circular and os-like projection into the bladder, and it gave the maximum of trouble, and he could not understand any more irregular form of growth to be met with. He did not believe in the efficacy of electricity, and when he found ro good results he stopped. He joined issue with Mr. Myles about syphon drainage. He thought the idea simply Utopian, although it might have a scientific basis. With regard to discerning between the membranous portion of the urethra and the bladder, they did not want that, because supra-pubic prostatectomy dealt with the prostate, and then by a perinæal incision into the membranous portion of the urethra it would meet the other finger, and a drainage tube could be inserted. Mr. Myles has denied the existence of a post-prostatic pouch, but he (Mr. Chance) thought that applied only to the healthy subject, because, as a 
matter of fact, he could put his finger into it in his last case. By residual urine he understood the water drawn off by the catheter after the patient bad passed as much as he was able. He said that even after emptying the bladder by the catheter, a considerable quantity still remains behind. His proof was that when they washed out the bladder and passed a catheter, and afterwards injected 14 ounces of water, the bladder was distended to considerably more than it should be by the 14 ounces. He referred to the advantages of the scoop, and said it was especially useful where they had only a small opening. 